\title{
Are radiation-induced cavernomas clinically relevant findings? Results from long-term follow-up with brain magnetic resonance imaging of childhood cancer survivors
}

\author{
Lucas Becker ${ }^{1}$, Judith Gebauer ${ }^{2,7}$, Jan Küchler ${ }^{3}$, Christian Staackmann ${ }^{4}$, Hannes Schacht ${ }^{1}$ \\ Melchior Lauten ${ }^{5}$, Ulf Jensen-Kondering ${ }^{6}$, Peter Schramm¹, Thorsten Langer ${ }^{5}$, \\ Alexander Neumann ${ }^{1}$

\begin{abstract}
1 Department of Neuroradiology, University Medical Center Schleswig-Holstein, Luebeck, Germany
2 Department of Internal Medicine I, University Medical Center Schleswig-Holstein, Luebeck, Germany

${ }^{3}$ Department of Neurosurgery, University Medical Center Schleswig-Holstein, Luebeck, Germany

${ }^{4}$ Department of Radiation Oncology, University Medical Center Schleswig-Holstein, Luebeck, Germany

${ }^{5}$ Department of Pediatric Oncology and Hematology, University Medical Center Schleswig-Holstein, Luebeck, Germany

${ }^{6}$ Department of Radiology and Neuroradiology, University Medical Center Schleswig-Holstein, Kiel, Germany

${ }^{7}$ Institute for Endocrinology and Diabetes, University of Luebeck, Germany
\end{abstract}

Radiol Oncol 2021; 55(3): 274-283.

Received 25 January 2021

Accepted 20 June 2021

Correspondance to: Alexander Neumann, MD, Department of Neuroradiology, University Medical Center Schleswig-Holstein, Ratzeburger Allee 160, 23538 Luebeck, Germany. E-mail: alexander.neumann@uksh.de

Lucas Becker and Judith Gebauer contributed equally and shared first authorship.

Disclosure: No potential conflicts of interest were disclosed.

This is an open access article under the CC BY-NC-ND license (http://creativecommons.org/licenses/by-nc-nd/4.0/).

Introduction. Radiation-induced cavernomas (RIC) after cranial radiotherapy have an unknown risk of hemorrhage. Zabramski magnetic resonance imaging (MRI) classification is touted as being able to indicate non-radiationinduced cavernomas hemorrhage risk. The aim of our study was to assess the hemorrhage risk of RIC during long-term follow-up of childhood cancer survivors based on brain MRI examinations.

Patients and methods. We analyzed retrospectively long-term follow-up data of 36 childhood cancer survivors after initial diagnosis with acute leukemia $(n=18)$ or brain tumor $(n=18)$, all treated with cranial radiotherapy. Detected RIC in long-term follow-up brain MRI (1.5 or 3 Tesla) were classified following the Zabramski MRI classification and were categorized into "high" (Zabramski type I, II or V) or "low" (type III or IV) risk of hemorrhage.

Results. 18 patients (50\%) showed RIC with a significant relation to the original tumor entity $(p=0.023)$ and the cumulative radiation dose to the brain $(p=0.016)$ : all 9 childhood cancer survivors diagnosed with medulloblastoma developed RIC. We classified RIC in only $3 / 36$ childhood cancer survivors (8\%) (1 patient with acute lymphoblastic leukemia [Zabramski type II] and 2 patients with medulloblastoma [type I and type II]) as high risk for hemorrhage, the remaining RIC were classified as Zabramski type IV with low risk for hemorrhage. None of the childhood cancer survivors with RIC showed symptomatic hemorrhages.

Conclusions. RIC are common late effects in childhood cancer survivors treated with cranial radiotherapy affecting half of these patients. However, only a few RIC (occurring in $8 \%$ of all reviewed childhood cancer survivors) were classified as high risk for hemorrhage and none of the childhood cancer survivors with RIC developed symptomatic hemorrhages. Thus, we conclude that RIC are low-risk findings in brain MRI and the course is mainly benign.

Key words: childhood cancer survivors; cranial radiotherapy; cavernomas; hemorrhage; brain magnetic resonance imaging 


\section{Introduction}

In recent decades, the percentage of children who can be cured from cancer has increased steadily, resulting in a growing number of childhood cancer survivors worldwide. ${ }^{1}$ However, many of these childhood cancer survivors develop chronic health conditions emerging years to decades later as a consequence of cancer therapy. ${ }^{2}$ Compared with chemo- or immunotherapy, late effects of radiation often occur in the former treated field, leading to subsequent neoplasms. ${ }^{3}$ The cumulative radiation dose correlates with rates of subsequent tumors in the central nervous system (CNS) that include mostly meningiomas or gliomas. ${ }^{4}$

Furthermore, cranial radiotherapy is known to increase the risk of de novo formation of cerebral cavernomas (syn. cavernous angiomas, cavernous hemangiomas, cavernous malformation) as well, which also occur sporadically or following a familial etiology and represent benign venous malformations with low flow and low pressure. ${ }^{5,6}$ However, while other radiation-induced neoplasms in most cases lead to rather clear-cut monitoring or therapy recommendations according to an interdisciplinary consensus, the handling approach for radiation-induced cavernomas (RIC) has yet to be defined, particularly in the context of the follow-up of childhood cancer survivors focused on here. Although the majority of cavernomas follow a complication-free course and do not require intervention, we are already aware of a tendency to symptomatic intracranial hemorrhage with potentially considerable clinical impact and even subsequent need for surgery. ${ }^{7-10}$ But so far systematic investigations concerning the hemorrhage risk of RIC, in particular, are not available.

Brain magnetic resonance imaging (MRI) is the modality of choice for the detection and follow-up imaging of cavernomas. ${ }^{11,12}$ In 1994, Zabramski et al. defined a grading system for familial cavernomas depending on MRI findings (Table 1$).{ }^{13}$ Nikoubashman et al. took up this classification in the recent past and investigated criteria to calculate prospective annual hemorrhage rates of cavernomas. ${ }^{14}$ The study showed high bleeding risks for Zabramski type I (29.8\%), type II (20.1\%) and a proposed new type $\mathrm{V}(23.1 \%)$ and low bleeding risks for type III and IV $(3.4 \%$ and $1.3 \%$ respectively). But in their study patients with a history of irradiation were explicitly excluded..$^{14}$

Thus it remains unclear whether Nikoubashman's interpretation of the MRI appearance of cavernomas based on the Zabramski classification might be helpful for estimation of the hemorrhage risk of RIC. We would like to help clarify this and therefore set our sights on assessing for the first time the risk of hemorrhage of RIC in the long-term follow-up of childhood cancer survivors with brain MRI in this case series.

\section{Patients and methods}

This study was approved by the Institutional Review Board of the University of Luebeck (14180, 18-087).

TABLE 1. MRI classification of cerebral cavernomas according to Zabramski et al.: type I, type II and an additionally proposed new type $V$ are associated with higher prospective hemorrhage rates, as published by Nikoubashman et al. in connection with non-radiation induced cavernomas ${ }^{13,14}$

\begin{tabular}{|c|c|c|}
\hline Lesion type & MRI signal characteristics & Pathological characteristics \\
\hline Type I & $\begin{array}{l}\text { T1: hyperintense core } \\
\text { T2: hyper- or hypointense core with surrounding } \\
\text { hypointense rim }\end{array}$ & $\begin{array}{l}\text { Subacute hemorrhage, surrounded by a rim of hemosiderin- } \\
\text { stained macrophages and gliotic brain }\end{array}$ \\
\hline Type II & $\begin{array}{l}\text { T1: reticulated mixed-signal core } \\
\text { T2: reticulated mixed-signal core with surrounding } \\
\text { hypointense rim }\end{array}$ & $\begin{array}{l}\text { Loculated areas of hemorrhage and thrombosis of varying ages, } \\
\text { surrounded by gliotic, hemosiderin-stained brain; in large lesions, } \\
\text { areas of calcification may be seen }\end{array}$ \\
\hline Type III & $\begin{array}{l}\text { T1: iso- or hypointense core } \\
\text { T2: hypointense with a hypointense rim that magnifies the size } \\
\text { of the lesion } \\
\text { GE: hypointense with greater magnification than T2 }\end{array}$ & $\begin{array}{l}\text { Chronic resolved hemorrhage, with hemosiderin staining within } \\
\text { and around the lesion }\end{array}$ \\
\hline Type IV & $\begin{array}{l}\text { T1: poorly seen or not visualized at all } \\
\text { T2: poorly seen or not visualized at all } \\
\text { GE: punctate hypointense lesions }\end{array}$ & $\begin{array}{l}\text { Two lesions in the category were pathologically documented as } \\
\text { telangiectasias }\end{array}$ \\
\hline Type V & $\begin{array}{l}\text { T1 and T2: visible parts in the center of the actual cavernoma; } \\
\text { the cavernoma is not fully distinguishable from hemorrhage }\end{array}$ & \\
\hline
\end{tabular}

GE $=$ gradient echo sequence 
252 patients in LTFU care in the late effects clinics in Luebeck (03/2014-02/2020)

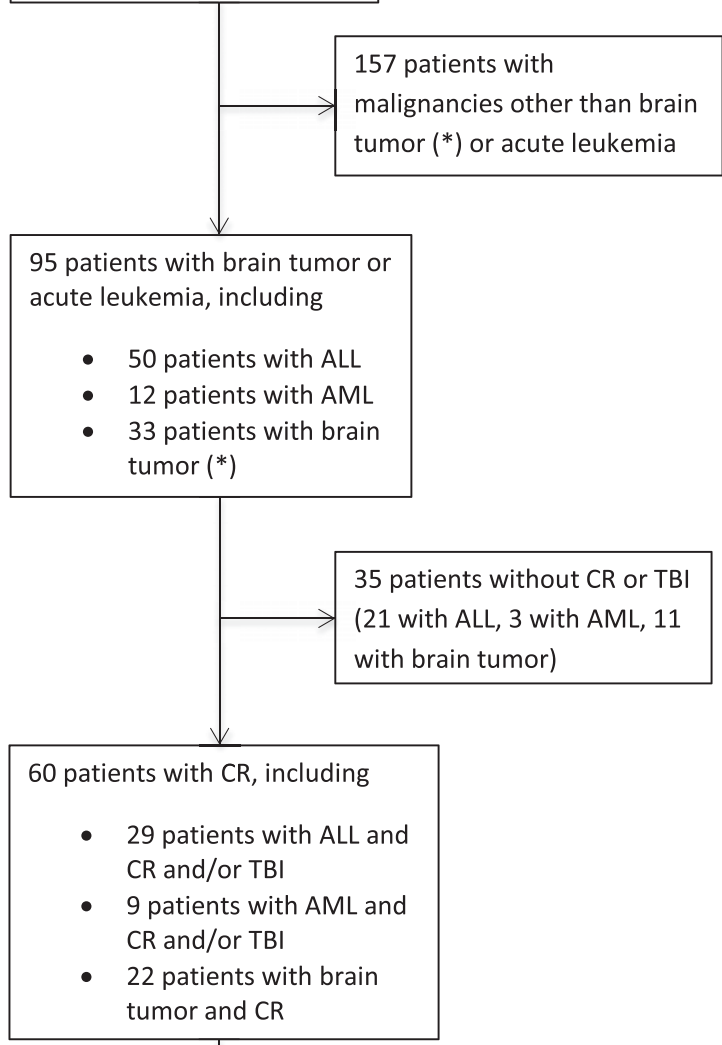

24 patients without brain MRI (16 with ALL, 4 with AML, 4 with brain tumor)

36 patients with $\geq 2$ brain $\mathrm{MRI}$ including

- 13 patients with ALL and $\mathrm{CR}$ and/or TBI

- 5 patients with AML and CR and/or TBI

- 18 patients with brain tumor and $C R$

FIGURE 1. Flowchart of the patient selection process.

* = craniopharyngeoma, ependymoma, germinoma, medulloblastoma $\mathrm{ALL}=$ acute lymphoblastic leukemia; $\mathrm{AML}$ = acute myeloid leukemia $\mathrm{CR}=$ cranial radiotherapy; $\mathrm{LTFU}=$ long-term follow-U; $M R I=$ magnetic resonance imaging; $\mathrm{TBI}=$ total body irradiation

\section{Patients}

We retrospectively analyzed 36 childhood cancer survivors out of 252 patients who were examined in the late effects clinic at the University Medical Center Schleswig Holstein in Luebeck over a pe- riod of 6 years (03/2014-02/2020). We limited the analysis to patients with acute leukemia or brain tumors who had been treated with cranial radiotherapy (alone or as part of total body irradiation) and had received cranial imaging as part of their follow-up. Additionally, at least 2 brain MRI examinations ( 1 at the time of the initial tumor diagnosis and at least 1 in the follow-up) were required for inclusion in the study. The patients at the late effects clinic (i) were younger than 18 at cancer diagnosis, (ii) were at least 18 at first follow-up in the clinic and iii) survived at least 5 years after the end of cancer therapy. Exclusion criteria for the study were (i) active malignancy and (ii) lack of consent. See the flowchart of the patient selection process in Figure 1.

\section{Magnetic resonance imaging of the brain including detection and classification of radiation-induced cavernomas}

All patients received cranial imaging with at least one follow-up MRI of the brain on 1.5 or 3 Tesla scanners. The MRI sequence protocol for detection and classification of RIC included a T1, T2 and T2*/susceptibility weighted imaging. Available brain MRI examinations were assessed and compared by two experienced neuroradiologists in consensual decisions. RIC were consensually detected and classified following the Zabramski classification and categorized into "high" ( $\geq 1$ type I and/or II and/or type V, in accordance with the proposal of Nikoubashman et al. ${ }^{14}$ ) or "low" (only type III and/or IV) risk of hemorrhage. Furthermore, other intracranial tumors as well as leukoencephalopathy and brain atrophy were analyzed. Leukoencephalopathy was graded into "mild" (focal lesions with hyper-intense signal in T2), "moderate" (confluent lesions) and "extensive" (diffuse lesions). Detected brain atrophy was divided semi-quantitatively into "mild", "moderate" and "severe".

\section{Statistical analysis}

Clinical and imaging data were collected in a standardized pseudonymized data file. Most data were analyzed in a descriptive manner. For statistical analysis we used the software IBM ${ }^{\circledR}$ SPSS $®$ (IBM Corp. Released 2016. IBM SPSS Statistics for Windows, Version 24.0. Armonk, NY: IBM Corp.). Categorical variables were analyzed in contingency tables using chi-squared test, or, if applicable, with the Fisher exact test. Mann-Whitney $U$ test was used to compare continuous variables. 
For the calculation of a possible association between the age at the initial tumor diagnosis (acute leukemia or brain tumor) and the occurrence of RIC the patients were dichotomized into the two groups "younger age" ( $\leq 8$ years) and "older age" ( $>8$ years) based on the median age calculated in this collective.

Statistical significance was accepted at $\mathrm{p}<0.05$.

\section{Results}

\section{Childhood cancer survivors}

The study group of childhood cancer survivors consisted of 36 patients with the diagnosis of acute leukemia or brain tumor. At the time of the initial cancer diagnosis their age ranged from 1 to 25 years (median 8 years, interquartile range [IQR] 5-14 years). There were 19 female (median 7 years, IQR 3-14 years) and 17 male (median 10 years, IQR 6-15 years). We found no significant difference in the age profile between the two gender groups $(p=0.505)$.

In particular, initial childhood cancer diagnoses were acute lymphoblastic leukemia (ALL) $(n=13)$, acute myeloid leukemia (AML) $(n=5)$, pilocytic astrocytoma $(n=2)$, intracranial germinoma $(n=$ $3)$, medulloblastoma $(n=9)$ and ependymoma ( $=3$ ) with need for cranial radiotherapy and polychemotherapy.

The median period of the last long-term followup examination in our late effects clinics after initial tumor diagnosis was 18 years (IQR 13-29).

\section{Cranial radiotherapy}

Cranial radiotherapy was applied in conventional fractionation for patients with ALL, AML, pilocytic astrocytoma and germinoma. However, in patients with medulloblastoma, cranial radiotherapy was given in conventional fractionation or as hyperfractionated treatment. Moreover, one patient with craniopharyngeoma was treated with proton therapy. The exact cumulative radiation dose to the brain could not be evaluated in this case. In patients with acute leukemia, total body irradiation consisted of 2 × 2.0 Gray (Gy) daily in three consecutive days. See Table 2 for scheme of cranial radiotherapy according to treatment protocols.

The median cumulative radiation dose in the 35 patients analyzed was 30.6 Gy (IQR 12-55 Gy). In all patients, no serious radiation-induced toxicities were reported. Individual's cumulative radiation doses to the brain are listed in Table 3.
TABLE 2. Cranial radiotherapy according to treatment protocols

\begin{tabular}{lll}
\hline Tumor entity & Dose/day & $\begin{array}{l}\text { Number of } \\
\text { fractions }\end{array}$ \\
\hline ALL & $1.5 \mathrm{~Gy}$ & $8,12,16,20$ or 26 \\
\hline AML & $1.5 \mathrm{~Gy}$ & 8 or 10 \\
\hline Pilocytic Astrocytoma & $1.8 \mathrm{~Gy}$ & 28 or 30 \\
\hline Germinoma & $1.6 \mathrm{~Gy}$ & 15 or 25 \\
\hline Medulloblastoma & $1.8 \mathrm{~Gy}$ & 30 or 34 \\
\hline
\end{tabular}

$\mathrm{ALL}=$ acute lymphoblastic leukemia; $\mathrm{AML}$ = acute myeloid leukemia; $\mathrm{Gy}$ = Gray

\section{Follow-up brain magnetic resonance imaging and findings of radiation- induced cavernomas}

Childhood cancer survivors received between 2 and 31 MRI examinations of the brain and we analyzed in total 383 MRI examinations. Radiation-induced meningiomas were found in four patients (11\%), one of whom had 2 and another had 5 meningiomas. Furthermore, in one patient we found a possible vestibular schwannoma, which, however, could not be reliably distinguished from an aneurysmal bone cyst via imaging. Gliomas were not detected in long-term follow-up. Leukoencephalopathies were found in 28 patients $(78 \%)$, while 12 of those $28(43 \%)$ were mild, 10 were moderate $(36 \%)$ and 6 extensive (21\%). According to semi-quantitatively analysis we found brain atrophy in 8 patients (22\%): Five of them mild (62.5\%) and three moderate $(36.5 \%)$, but detected no cases of severe brain atrophy.

For further long-term comorbidities see Table 3.

In long-term follow-up 162 of 383 brain MRI examinations $(42 \%)$ did not meet inclusion criteria because of lacking $\mathrm{T} 2 *$ susceptibility weighted imaging sequences or movement or other artifacts. Thus, we were able to analyze 221 brain MRI examinations (58\%) concerning RIC.

$18 / 36$ patients $(50 \%)$ showed RIC. We found a significant relation of existing RIC to the original tumor entity $(p=0.023)$ and to the cumulative radiation dose to the brain $(p=0.016)$.

The occurrence of RIC was equal in female $(\mathrm{n}=$ $9)$ and male patients $(n=9)(p=0.500)$. There was no significant association between the occurrence of RIC with the age at initial tumor diagnosis respectively start of cranial radiotherapy. Furthermore, we found no significant differences of RIC in the dichotomized patient groups "younger age" $(\leq 8$ years, RIC $\mathrm{n}=8$ ) and "older age" ( $>8$ years, RIC $\mathrm{n}=10)(\mathrm{p}=0.370)$. 
TABLE 3. Patient characteristics, therapy data, findings of radiation-induced cavernomas and other comorbidities

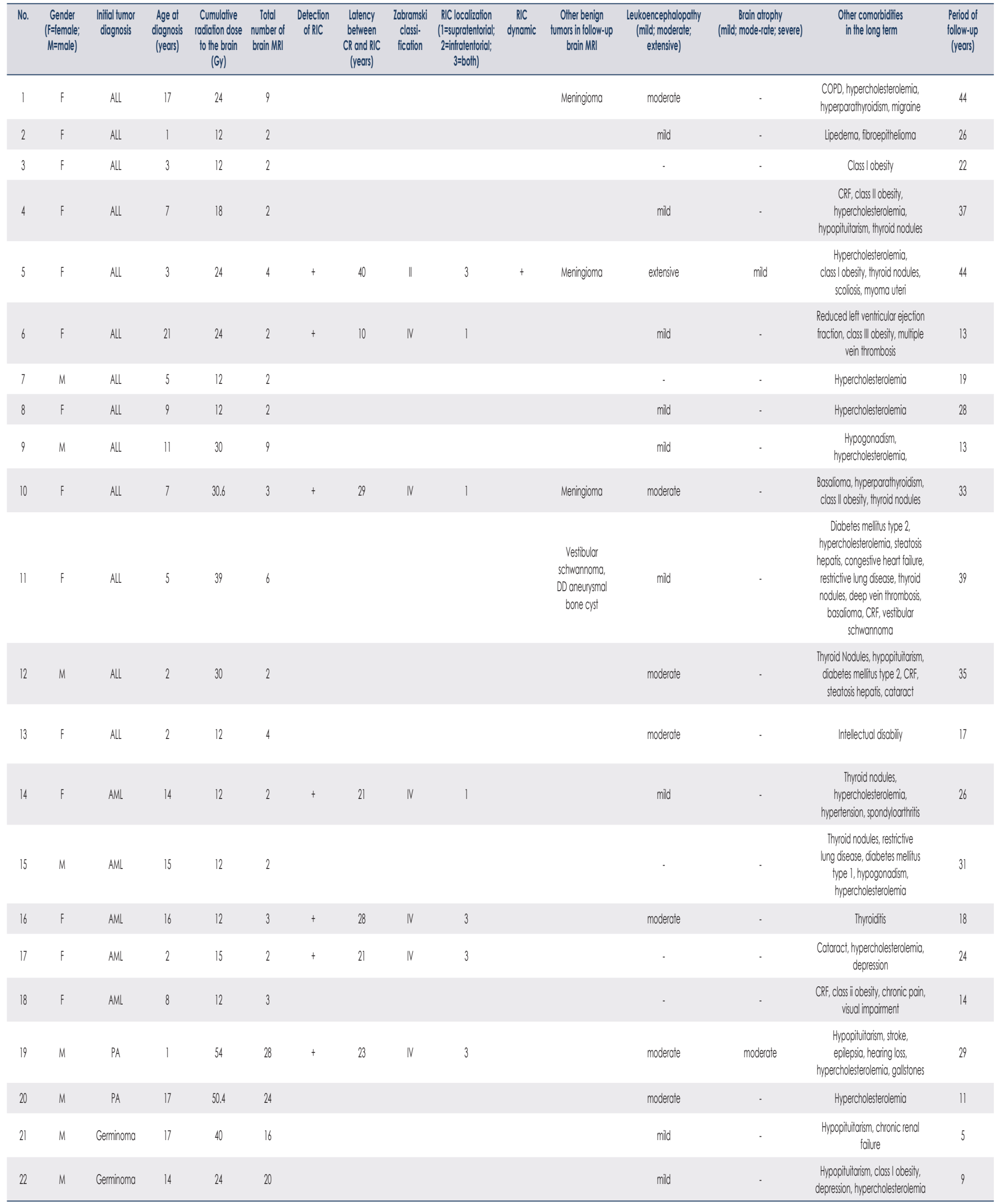




\begin{tabular}{|c|c|c|c|c|c|c|c|c|c|c|c|c|c|c|c|}
\hline No. & $\begin{array}{c}\text { Gender } \\
\text { (F=female; } \\
\text { M=mole) }\end{array}$ & $\begin{array}{l}\text { Initifal lumor } \\
\text { diagnosis }\end{array}$ & $\begin{array}{l}\text { Age of } \\
\text { diagnosis } \\
\text { (years) }\end{array}$ & $\begin{array}{l}\text { Cumulative } \\
\text { radiation dose } \\
\text { to the brain } \\
\text { (Gy) }\end{array}$ & $\begin{array}{c}\text { Iotal } \\
\text { number of } \\
\text { brain MRI }\end{array}$ & $\begin{array}{l}\text { Detection } \\
\text { of RlC }\end{array}$ & $\begin{array}{l}\text { latency } \\
\text { between } \\
\text { CR and RIC } \\
\text { (years) }\end{array}$ & $\begin{array}{l}\text { Zabramski } \\
\text { classi- } \\
\text { fication }\end{array}$ & $\begin{array}{l}\text { RIC localization } \\
\text { (1=supratentorial; } \\
\text { 2=intratentorial; } \\
\text { 3=both) }\end{array}$ & $\begin{array}{l}\text { RIC } \\
\text { dynamic }\end{array}$ & $\begin{array}{l}\text { Other benign } \\
\text { tumors in follow-vp } \\
\text { brain MRI }\end{array}$ & $\begin{array}{l}\text { Leukoencephalopothy } \\
\text { (mild; moderate; } \\
\text { exiensive) }\end{array}$ & $\begin{array}{c}\text { Brain atrophy } \\
\text { (mild; mode-rate; severe) }\end{array}$ & $\begin{array}{l}\text { Other comorbidilies } \\
\text { in the long term }\end{array}$ & $\begin{array}{l}\text { Period of } \\
\text { follow-up } \\
\text { (years) }\end{array}$ \\
\hline 24 & M & $C P$ & 17 & n.a. & 15 & & & & & & & mild & . & $\begin{array}{l}\text { Hypopitiutaraism, class I } \\
\text { obesity, hypercholesterolemia, } \\
\text { autoimmune polyendocine } \\
\text { syndrome type } 2 \text { with diabetes } \\
\text { mellitus type I, vitiligo, thyroidilis }\end{array}$ & 13 \\
\hline 25 & $F$ & $\begin{array}{l}\text { Medullo- } \\
\text { blastoma }\end{array}$ & 7 & 55 & 3 & + & 27 & IV & 2 & & Meningioma & & mild & $\begin{array}{l}\text { Hypothyrodism, thyroid nodules, } \\
\text { hearing loss, ashmma }\end{array}$ & 28 \\
\hline 26 & M & $\begin{array}{l}\text { Medullo- } \\
\text { blastoma }\end{array}$ & 8 & 54 & 25 & + & 5 & IV & 3 & + & & mild & . & Hypopituitarism, hearing loss & 14 \\
\hline 27 & M & $\begin{array}{l}\text { Medullo- } \\
\text { blastoma }\end{array}$ & 8 & 54 & 21 & + & 2 & 1 & 3 & + & & moderate & moderate & Hypopituitarism, hearing loss & 10 \\
\hline 29 & M & $\begin{array}{l}\text { Medullo- } \\
\text { blastoma }\end{array}$ & 11 & 68.6 & 24 & + & 2 & IV & 3 & + & & . & mild & $\begin{array}{l}\text { Spinal hygroma, hypothyrodism, } \\
\text { growth hormone deliciency }\end{array}$ & 14 \\
\hline 30 & M & $\begin{array}{l}\text { Medullo- } \\
\text { blastoma }\end{array}$ & 6 & 68.6 & 18 & + & 7 & IV & 3 & + & & . & . & $\begin{array}{l}\text { Hypopituitaraism, hearing loss, } \\
\text { intellectual disability }\end{array}$ & 19 \\
\hline 31 & M & $\begin{array}{l}\text { Medullo- } \\
\text { blastoma }\end{array}$ & 9 & 60 & 3 & + & 29 & $\|$ & 2 & & & mild & & $\begin{array}{l}\text { Basalioma, heaing loss, } \\
\text { intellectual disobility }\end{array}$ & 30 \\
\hline 32 & $\mathrm{~F}$ & $\begin{array}{l}\text { Medullo- } \\
\text { blastoma }\end{array}$ & 25 & 60 & 6 & + & 18 & IV & 3 & & & extensive & moderate & $\begin{array}{l}\text { Coxathrosis with total } \\
\text { endoprothessis, gonarthosis }\end{array}$ & 18 \\
\hline 33 & M & $\begin{array}{l}\text { Medullo- } \\
\text { blastoma }\end{array}$ & 2 & 60 & 13 & + & 7 & IV & 3 & & & moderate & mild & $\begin{array}{l}\text { Visual Impaiment, groth hormone } \\
\text { deficiency, hypothyroidism, } \\
\text { intelectual disability, hearing loss, } \\
\text { steatossis hepatis }\end{array}$ & 17 \\
\hline
\end{tabular}

$\mathrm{ALL}=$ acute lymphoblastic leukemia; $\mathrm{AML}=$ acute myeloid leukemia; $\mathrm{CRF}=$ cancer related fatigue; $\mathrm{COPD}=$ chronic obstructive pulmonary disease; $\mathrm{CP}=\mathrm{craniopharyngeoma;}$ $\mathrm{CR}=$ cranial radiotherapy; $\mathrm{DD}=$ differential diagnosis; $\mathrm{MRI}=$ magnetic resonance imaging; $\mathrm{n} . \mathrm{a}$. = not available; $\mathrm{PA}=$ pilocytic astrocytoma; $\mathrm{PCHT}=$ polychemotherapy; RIC = radiation-induced cavernomas

The cumulative radiation dose differed significantly between childhood cancer survivors with RIC (median 54 Gy [IQR 24-60 Gy]) and childhood cancer survivors without RIC (median 24 Gy [IQR $12-40 \mathrm{~Gy}])(\mathrm{p}=0.019)$.

All 9 childhood cancer survivors with treated medulloblastoma developed RIC (100\%). Occurrences of RIC dependent on the other tumor entities were: ALL 3/13 (23\%), AML 3/5 (60\%), pilocytic astrocytoma $1 / 2(50 \%)$, germinoma $1 / 3(33 \%)$ and ependymoma $1 / 3(33 \%)$. One patient with craniopharyngeoma did not develop RIC. We classified RIC only in 1 patient $(3 \%)$ with ALL (Zabramski type II) and in 2 patients $(6 \%)$ with medulloblastoma (Zabramski type I and type II) as high risk for hemorrhage while all other detected RIC were classified as Zabramski type IV with low risk for hemorrhage. We did not find any statistically significant difference between RCI with low and high risk for hemorrhage depending on the original tumor entity $(p=0.737)$. We did not categorize RIC as Zabramski type III or V, in accordance with the proposal of Nikoubashman et al. ${ }^{14}$ Thus, only $3 / 18$ childhood cancer survivors with RIC $(17 \%)$ and $3(8 \%)$ of all reviewed childhood cancer survivors with at least 2 brain MRI examinations developed lesions with a high risk for hemorrhage. As a main result of the study none of the childhood cancer survivors with RIC showed symptomatic intracranial hemorrhages. The localization of RIC was only supratentorial in 15 of the 36 childhood cancer 

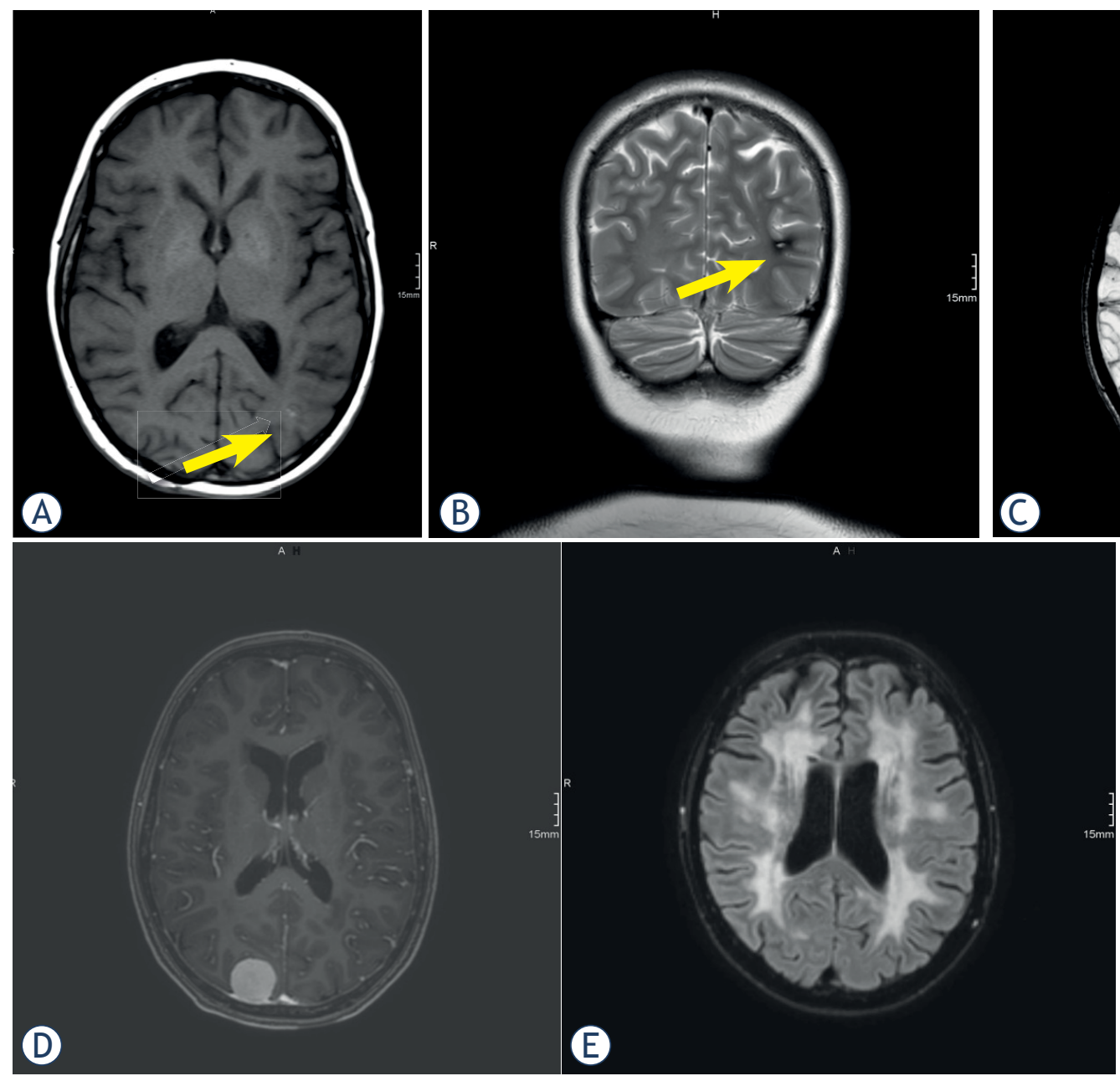

FIGURE 2. Examples of radiationinduced cavernomas with a type II lesion (A, B) and multiple type IV lesions (C) according to the Zabramski classification. Radiationinduced meningioma (D) and leukoencephalopathy with brain atrophy (E). survivors (42\%), in two childhood cancer survivors only infratentorial (5\%) and in 19 childhood cancer survivors both supra- and infratentorial (53\%). RIC occurred after cranial radiotherapy in a median time period of 15.5 years (IQR 5-27). Six of the 36 childhood cancer survivors (17\%) showed dynamic findings of RIC, and there were changes in the number and/or size of individual RIC without any accompanying changes in the Zabramski classification. Three childhood cancer survivors (8\%) developed only a single RIC documented in brain MRI. Table 3 shows the summarized results. Examples of brain magnetic resonance imaging from childhood cancer survivors during long long-term follow-up are shown in Figure 2.

\section{Discussion}

The aim of this study was to describe for the first time the course of RIC during long-term follow- up of childhood cancer survivors, those formerly treated with cranial radiotherapy in connection with the initial diagnosis of leukemia or brain tumor. We thus assessed the hemorrhage risk of RIC following Nikoubashman's interpretation of the MRI appearance of cavernomas based on the Zabramski classification and examined the question of how to deal with RIC that frequently appear in MRI follow-up examinations of childhood cancer survivors. In our experience to date this seems to be distinctly more difficult than providing recommendations regarding extraaxial or intraaxial neoplasms (especially meningiomas or gliomas), which have already been the subject of extensive discussion in relevant literature as long-term consequences. ${ }^{4}$ Several guidelines, often based on a risk stratification system mainly taking into account the initial cancer diagnosis and the treatment received, recommend specific follow-up examinations to facilitate early diagnosis and treatment of these sequelae. ${ }^{15}$ 
Besides neoplasms, neurocognitive deficits in connection with pathological findings in brain MRI, such as leukoencephalopathy or brain atrophy, are reported in connection with childhood cancer survivors. ${ }^{16}$ Also our results showed 4 radiation-induced meningiomas, but no gliomas. There were variable findings of leukoencephalopathy, brain atrophy and several other comorbidities, though this was not a focal point of our studies.

Our study focuses on RIC. An assumed correlation between cranial radiotherapy and cavernomas in children was primarily described in $1994 .{ }^{17}$ In the following years this phenomenon was investigated extensively in several studies and summarized in review articles with a visible predominance in young patients treated for cancer. ${ }^{18-24}$ Furthermore, researchers also looked into the question of whether RIC differ from other, sporadically or familially occurring cavernomas regarding their natural course. Baumgartner $e t$ al. showed findings after irradiation radiologically and pathologically similar to sporadically occurring cavernomas. ${ }^{25}$ Cha et al., on the other hand, found differences in histological findings in a comparison between 10 patients with "de novo" cavernomas and 7 patients with a total of 8 RIC and regarded the findings after radiation more as inactive organizing hematomas in blood-filled spaces after tissue destruction and thus different from proliferation of malformed vasculature in sporadic cavernomas. ${ }^{26}$ According to a study with a specific histopathological focus based on 13 patients with RIC, there is, both histologically and with regard to immunohistochemistry, an overlap to non-radiationinduced cavernomas (5/13) as well as to organizing coagulum-like lesions (8/13), as described by Cha et al., so that one must assume two different etiological mechanisms in the pathogenesis. ${ }^{27}$

Concerning RIC, our results include a significant relation to the initial tumor entity and the cumulative radiation dose to the brain, as reflected by the occurrence of RIC without exception in patients treated for medulloblastoma with high cumulative radiation doses. In 2007 Lew et al. looked at precisely such patients who had received radiation therapy for medulloblastomas in childhood and described cavernomas in this case as common with an increasing incidence over time. ${ }^{28}$ They concluded that most of these lesions follow a benign course and do not require intervention. ${ }^{28}$ Furthermore, results that show a correlation between RIC and the cumulative radiation dose can be found in a more recent study focusing on adult cancer patients. ${ }^{29}$

With regard to the correlation between RIC and cranial radiotherapy, data on the time lag of the oc- currence after radiation are additionally available. Following examination of 5 patients with cavernomas after cranial or craniospinal irradiation, Jain et al. described a latency interval between treatment and time of detection of cavernomas with a mean of 19.5 years. ${ }^{5}$ The latency that we found between cranial radiotherapy and detection of RIC with a median time period of 15.5 years matches these investigations (IQR 5-27). Moreover, Strenger et al. has published results that also focus on risk factors for the occurrence of RIC. ${ }^{30}$ The investigations encompassed 171 patients and showed that particularly children $<10$ years of age at the time of irradiation have a higher risk for RIC, whose occurrence was documented here as taking place in a period of 2.9-18.4 years after radiation..$^{30}$ Singla et al. examined the long-term course of irradiated patients and described for all of them an occurrence of cavernomas with a latency of 2-8 years - though only 5 patients with ALL were analyzed. ${ }^{31}$

Due to possible hemorrhage complications of RIC, the two latter studies very explicitly advocated imaging monitoring and Singla et al. described neurological symptoms and individual craniotomies because of RIC in 4 of 5 patients. ${ }^{30,31}$ We did not document any symptomatic hemorrhages in connection with RIC in our patient group.

A number of studies have examined the hemorrhage risk of cavernomas in general. Flemming et al. calculated a prospective risk in this context in a group of 292 patients with cavernomas and consecutive hemorrhage. ${ }^{7}$ It was determined that prior hemorrhage is one of the factors that may predict future hemorrhage. ${ }^{7}$ One publication showed in a comparison of the features of RIC (32 patients) with other cavernomas (272 patients) no differences in symptomatic hemorrhages. ${ }^{32}$ However, here RIC proved to be more likely to occur multilocular. ${ }^{32}$ This matches our results, which predominantly showed multilocular and small lesions (Zabramski type IV). In particular, Nikoubashman et al. investigated those small, dot-like cavernomas in another study from 2013.33 It shows a low bleeding rate of $0.7 \%$ per lesion-year according to a survey specifically of 18 children. ${ }^{33}$ Nonetheless, it was propagated that even those small cavernomas are not purely benign lesions. ${ }^{33}$

It is certainly more difficult, however, to deal with lesions classified as Zabramski type I or II. As a reminder: type I is a (sub-)acute hemorrhage while type II as a "popcorn lesion" shows multiple hemorrhages. Here we again refer to data on the generally increased bleeding risk of cavernomas with already existing hemorrhage. ${ }^{7}$ Among others, 
Jeon et al. based their study of 326 adults with cavernomas, like us, on the Zabramski classification. ${ }^{34}$ The study showed that not only prior symptomatic hemorrhage, but also MRI appearance could be related to prospective symptomatic hemorrhages of cavernomas. ${ }^{34}$ The bleeding risk of cavernomas was also examined in a study by Al-Shahi Salman et al., which additionally evaluated gender-specific differences. ${ }^{35}$ This study found that the risk of recurrent hemorrhage is greater than the risk of a first bleeding event and greater for women than for men. ${ }^{35}$

As regards our results, we classified RIC as high risk for hemorrhage (Zabramski type I and II) in only 3 of 36 childhood cancer survivors (8\%) and all other RIC were classified as low-risk lesions (Zabramski type IV [microhemorrhagic]). In this risk assessment we orient ourselves to the proposal made by Nikoubashman et al. ${ }^{14}$ After analysis of 199 MRI examinations in total with 1558 distinct lesions this team depicted 5 different types of cavernomas via 1.5T MRI (T1, T2 and T2*) while using a three-tier classification shown as graphic illustrations and corresponding MRI findings. It turned out that the cavernomas described as Zabramski type I, II and an additionally proposed new type $\mathrm{V}$ (with visible parts in the center of the actual cavernoma) had a significantly higher annual hemorrhage rate than type III and IV lesions. ${ }^{14}$ Thus, the presence of acute or subacute blood degradation was statistically the strongest indicator for an increased hemorrhage risk with the conclusion that it is possible to predict hemorrhage rates based on the Zabramski classification. ${ }^{14}$ As mentioned previously, these results were not related to RIC. Nevertheless, just in connection with RIC we consider the initial application of Nikoubashman's interpretation of the MRI appearance of cavernomas based on the Zabramski classification for an assessment of the hemorrhage risk to be a very meaningful approach.

It must be emphasized that in this retrospective case series half of the childhood cancer survivors examined in long-term follow-up showed RIC in follow-up brain MRI. Thus, our study supports the development of cavernomas after cranial radiotherapy as a common side effect in childhood cancer survivors, though most RIC are not likely to hemorrhage and are detected incidentally. Our results therefore suggest a mainly benign course of RIC. Consequently we conclude that the complication risk of RIC can definitely be described in good conscience as low in communication with childhood cancer survivors as long as one applies assessments of existing classifications. ${ }^{13,14}$ This ensures an advantageous aspect in dealing with patients who might feel anxious as a result of their prior cancer history. Studies with larger groups of patients and stronger study designs may possibly clarify whether dealing with RIC should be included and defined in the guidelines in connection with childhood cancer survivors.

Thus, we conclude that RIC are common late effects in childhood cancer survivors treated with cranial radiotherapy affecting half of the patients. However, only a few RIC (occurring in $8 \%$ of all reviewed childhood cancer survivors) were classified as high risk for hemorrhage and the majority of RIC follow a benign course. None of the childhood cancer survivors with RIC developed symptomatic hemorrhages. These results augment accurate risk assessment in individualized long-term follow-up also in order to reduce morbidity in childhood cancer survivors. In this context we are addressing an important point in the communication with childhood cancer survivors.

\section{Limitations}

A retrospective approach with a relatively small sample size is a limitation of the present analysis. But, in particular, the accumulation of data concerning childhood cancer survivors also in the long-term follow-up, focused on here, is extremely rare, also in university hospitals. Nevertheless we strongly believe that this to our knowledge first study of this specific topic is valuable to assess the clinical relevance of radiation-induced cavernomas in brain MRI and add to be clear about their low risk for hemorrhage (with importance for patients and for physicians).

We are also restricted by the selection bias concerning inconsistent brain MRI data with lack of standardized imaging protocols in many MRI examinations conducted abroad and in some cases only a single follow-up examination with an extensive time lag until radiation. Furthermore, we did not focus on a detailed description of the irradiated fields, in particular, and the localization of RIC in our patient group, but we propose that this topic should be assessed after examination of larger collectives. The correlation between RIC and cranial radiotherapy that we assume in our results naturally remains unproven, also histologically, in the end (in this connection, the differential diagnoses cerebral microhemorrhages or teleangiectasias also have to be considered). The extent to which hemorrhages from cavernomas correlate with clinical 
symptoms is a complex issue of its own that this study cannot resolve on the basis of a relatively small group without symptomatic hemorrhages.

\section{References}

1. Miller KD, Nogueira L, Mariotto AB, Rowland JH, Yabroff KR, Alfano CM, et al. Cancer treatment and survivorship statistics, 2019. CA Cancer J Clin 2019; 69: 363-85. doi: 10.3322/caac.21565

2. Oeffinger KC, Mertens AC, Sklar CA, Kawashima T, Hudson MM, Meadows AT, et al. Chronic health conditions in adult survivors of childhood cancer. $N$ Engl J Med 2006; 355: 1572-82. doi: 10.1056/NEJMsa060185

3. Armstrong GT, Stovall M, Robison LL. Long-term effects of radiation exposure among adult survivors of childhood cancer: results from the childhood cancer survivor study. Radiat Res 2010; 174: 840-50. doi: 10.1667/RR1903.1

4. Bowers DC, Nathan PC, Constine L, Woodman C, Bhatia S, Keller K, et al. Subsequent neoplasms of the CNS among survivors of childhood cancer: a systematic review. Lancet Oncol 2013; 14: e321-8. doi: 10.1016/S14702045(13)70107-4

5. Jain R, Robertson PL, Gandhi D, Gujar SK, Muraszko KM, Gebarski S. Radiation-induced cavernomas of the brain. AJNR Am J Neuroradiol 2005; 26: $1158-62$.

6. Hoz SS. Cavernoma and other malformations. In: Hoz SS, editor. Vascular neurosurgery. Berlin: Springer, Cham. 2017. p.p. 137-47. doi: 10.1007/9783-319-49187-5_4

7. Flemming KD, Link MJ, Christianson TJ, Brown RD Jr. Prospective hemorrhage risk of intracerebral cavernous malformations. Neurology 2012; 78: 632-6. doi: 10.1212/WNL.0b013e318248de9b

8. Consales A, Piatelli G, Ravegnani M, Pavanello M, Striano P, Zoli ML, et al. Treatment and outcome of children with cerebral cavernomas: a survey on 32 patients. Neurol Sci 2010; 31: 117-23. doi: 10.1007/s10072-009-0157-0

9. Giulioni M, Acciari N, Padovani R, Galassi E. Results of surgery in children with cerebral cavernous angiomas causing epilepsy. Br J Neurosurg 1995; 9:135-41. doi: 10.1080/02688699550041467

10. Acciari N, Galassi E, Giulioni M, Pozzati E, Grasso V, Palandri G, et al. Cavernous malformations of the central nervous system in the pediatric age group. Pediatric Neurosurgery 2009; 45: 81-104. doi: 10.1159/000209283

11. Hegde AN, Mohan S, Lim CC. CNS cavernous haemangioma: "popcorn" in the brain and spinal cord. Clin Radiol 2012; 67: 380-8. doi: 10.1016/j. crad.2011.10.013

12. Awad IA, Polster SP. Cavernous angiomas: deconstructing a neurosurgical disease. J Neurosurg 2019; 131: 1-13. doi: 10.3171/2019.3.JNS181724

13. Zabramski JM, Wascher TM, Spetzler RF, Johnson B, Golfinos J, Drayer BP, et al. The natural history of familial cavernous malformations: results of an ongoing study. J Neurosurg 1994; 80: 422-32. doi: 10.3171/jns.1994.80.3.0422

14. Nikoubashman O, Rocco FD, Davagnanam I, Mankad K, Zerah M, Wiesmann $M$. Prospective hemorrhage rates of cerebral cavernous malformations in children and adolescents based on MRI appearance. AJNR Am J Neuroradiol 2015; 36: 2177-83. doi: 10.3174/ajnr.A4427

15. Children's Oncology Group. Long-term follow-up guidelines for survivors of childhood, adolescent, and young adult cancer. Version 4.0, October 2013. [cited 2021-01-10]. Available at: www.survivorshipguidelines.org/ pdf/Itfuguidelines_40.pdf

16. Cheung YT, Sabin ND, Reddick WE, Bhojwani D, Liu W, Brinkman TM, et al. Leukoencephalopathy and long-term neurobehavioural, neurocognitive, and brain imaging outcomes in survivors of childhood acute lymphoblastic leukaemia treated with chemotherapy: a longitudinal analysis. Lancet Haematol 2016; 3: e456-e66. doi: 10.1016/S2352-3026(16)30110-7

17. Ciricillo SF, Cogen PH, Edwards MS. Pediatric cryptic vascular malformations: presentation, diagnosis and treatment. Pediatr Neurosurg 1994; 20: 137-47. doi: 10.1159/000120776

18. Nimjee SM, Powers CJ, Bulsara KR. Review of the literature on de novo formation of cavernous malformations of the central nervous system after radiation therapy. Neurosurgical Focus 2006; 21: 1-6. doi: 10.3171/ foc.2006.21.1.5
19. Di Giannatale A, Morana G, Rossi A, Cama A, Bertoluzzo L, Barra S, et al. Natural history of cavernous malformations in children with brain tumors treated with radiotherapy and chemotherapy. J Neurooncol 2014; 117: 31120. doi: 10.1007/s11060-014-1390-9

20. Baumgartner JE, Ater JL, Ha CS, Kuttesch JF, Leeds NE, Fuller GN, et al. Pathologically proven cavernous angiomas of the brain following radiation therapy for pediatric brain tumors. Pediatr Neurosurg 2003; 39: 201-7. doi: $10.1159 / 000072472$

21. Heckl S, Aschoff A, Kunze S. Radiation-induced cavernous hemangiomas of the brain. Cancer 2002; 94: 3285-3291. doi: 10.1002/cncr.10596

22. Lena G, Ternier J, Paz-Paredes A, Scavarda D. Central nervous system cavernomas in children. Neurochirurgie 2007; 53: 223-37. doi: 10.1016/j. neuchi.2007.02.011

23. Burn S, Gunny R, Phipps K, Gaze M, Hayward R. Incidence of cavernoma development in children after radiotherapy for brain tumors. J Neurosurg 2007; 106: 379-83. doi: 10.3171/ped.2007.106.5.379

24. Mottolese C, Hermier M, Stan H, Jouvet A, Saint-Pierre G, Froment JC, et al. Central nervous system cavernomas in the pediatric age group. Neurosurg Rev 2001; 24: 55-71. doi: 10.1007/pl00014581

25. Baumgartner JE, Ater JL, Ha CS, Kuttesch JF, Leeds NE, Fuller GN, et al. Pathologically proven cavernous angiomas of the brain following radiation therapy for pediatric brain tumors. Pediatr Neurosurg 2003; 39: 201-7. doi: 10.1159/000072472

26. Cha YJ, Nahm JH, Ko JE, Shin HJ, Chang J-H, Cho NH, et al. Pathological evaluation of radiation-induced vascular lesions of the brain: Distinct from de novo cavernous hemangioma. Yonsei Med J 2015; 56: 1714-20. doi: 10.3349/ymj.2015.56.6.1714

27. Kleinschmidt-DeMasters BK, Lillehei KO. Radiation-induced cerebral vascular "Malformations" at biopsy. J Neuropathol Exp Neurol 2016; 75: 1081-92. doi: 10.1093/jnen/nlw085

28. Lew SM, Morgan JN, Psaty E, Lefton DR, Allen JC, Abbott R. Cumulative incidence of radiation-induced cavernomas in long-term survivors of medulloblastoma. J Neurosurg 2006; 104: 103-7. doi: 10.3171/ped.2006.104.2.103

29. Cacho-Díaz B, Salmerón-Moreno K, Lorenzana-Mendoza N, Reyes A, ValdésFerrer SI, Gómez-Ahumada G, et al. Radiotherapy induced cavernomas in adult cancer patients. Radiother Oncol 2018; 127: 287-291. doi: 10.1016/j. radonc.2018.02.026

30. Strenger V, Sovinz P, Lackner H, Dornbusch HJ, Lingitz H, Eder HG, et al. Intracerebral cavernous hemangioma after cranial irradiation in childhood. Incidence and risk factors. Strahlenther Onkol 2008; 184: 276-80. doi: 10.1007/s00066-008-1817-3

31. Singla A, Brace O'Neill J, Smith E, Scott RM. Cavernous malformations of the brain after treatment for acute lymphocytic leukemia: presentation and long-term follow-up. J Neurosurg Pediatr 2013; 11:127-32. doi: 10.3171/2012.11.PEDS12235

32. Cutsforth-Gregory JK, Lanzino G, Link MJ, Brown Jr RD, Flemming KD. Characterization of radiation-induced cavernous malformations and comparison with a nonradiation cavernous malformation cohort. J Neurosurg 2015; 122: 1214-22. doi: 10.3171/2015.1.JNS141452

33. Nikoubashman O, Wiesmann M, Tournier-Lasserve E, Mankad K, Bourgeois $\mathrm{M}$, Brunelle $\mathrm{F}$, et al. Natural history of cerebral dot-like cavernomas. Clin Radiol 2013; 68: e453-59. doi: 10.1016/j.crad.2013.02.010

34. Jeon JS, Kim JE, Chung YS, Oh S, Ahn JH, Cho WS, et al. A risk factor analysis of prospective symptomatic haemorrhage in adult patients with cerebral cavernous malformation. J Neurol Neurosurg Psych 2014; 85: 1366-70. doi: 10.1136/jnnp-2013-306844

35. Al-Shahi Salman R, Hall JM, Horne MA, Moultrie F, Josephson CB, Bhattacharya JJ, et al. Untreated clinical course of cerebral cavernous malformations: a prospective, population-based cohort study. Lancet Neurol 2012; 11: 217-24. doi: 10.1016/S1474-4422(12)70004-2 